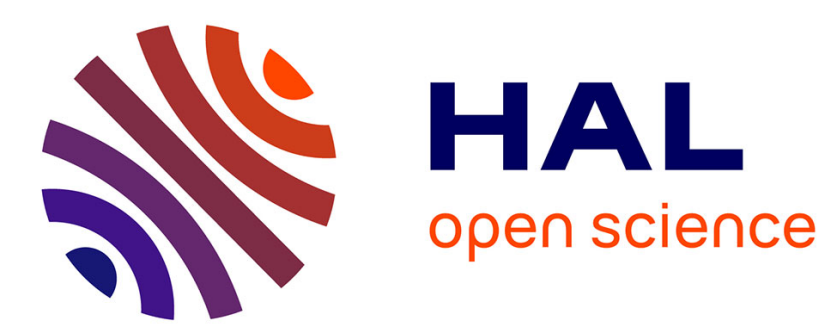

\title{
Recristallisation Statique et Croissance de Grain d'un Acier Austénitique Inoxydable
}

\author{
H. Tsukahara, A.-M. Chaze, Christophe Levaillant, S. Hollard
}

\section{To cite this version:}

H. Tsukahara, A.-M. Chaze, Christophe Levaillant, S. Hollard. Recristallisation Statique et Croissance de Grain d'un Acier Austénitique Inoxydable. J. Phys. IV, 1995, 05 (C3), p. 29-37. 10.1051/jp4:1995303 . jpa-00253669

\section{HAL Id: jpa-00253669 https://hal.science/jpa-00253669}

Submitted on 1 Jan 1995

HAL is a multi-disciplinary open access archive for the deposit and dissemination of scientific research documents, whether they are published or not. The documents may come from teaching and research institutions in France or abroad, or from public or private research centers.
L'archive ouverte pluridisciplinaire HAL, est destinée au dépôt et à la diffusion de documents scientifiques de niveau recherche, publiés ou non, émanant des établissements d'enseignement et de recherche français ou étrangers, des laboratoires publics ou privés. 


\title{
Recristallisation Statique et Croissance de Grain d'un Acier Austénitique Inoxydable
}

\author{
H. Tsukahara, A.-M. Chaze, C. Levaillant*, et S. Hollard** \\ Ecole des Mines de Paris, CEMEF, B.P. 207, 06904 Sophia-Antipolis, France \\ * Ecole des Mines d'Albi Carmaux, 81013 Albi CT cedex, France \\ ** FORTECH, B.P. 173, 09102 Pamiers cedex, France
}

\begin{abstract}
Static Recrystallization (SR), is here used as a way to get an ASTM [1] grain size of 3 or so. Making a prediction of the effect of both deformation and temperature on the kinetics of the SR phenomena is the first step toward a global microstructure prediction at the end of the manufacturing sequence. It is the main goal of the study here after described. In this study, we did hot compression tests $\left(950-1200^{\circ} \mathrm{C}\right)$ and analyzed them in terms of recrystallized fraction (quantitative metallography) using to classic Avrami equation. We observed some time exponents $(k)$ smaller than 1 , which goes against Avrami's theory, which only describes equal or above 1 exponents. The time for half recrystallization $\left(t_{0.5}\right)$ has also been determined, according to C.M. Sellars method and reached a satisfactory, experiment-calculation agreement.

After completion of the recrystallization, the Grain Growth phenomenon occurs. Thanks to a wide study of thermal annealings, we found a $A=A_{0}+\alpha \cdot t^{n}$ type of equation, where $\alpha$ is a variable involving $Q$, an activation energy, through an Arrhenius equation.
\end{abstract}

\section{INTRODUCTION}

Lors de procédés de déformation à chaud $\left(\approx 1050^{\circ} \mathrm{C}\right)$ tels que le forgeage, la structure interne du matériau, ou microstructure, se modifie tout autant qu'entre les différentes passes lorsque le métal est maintenu à température élevée. La Recristallisation Statique (RXS) est importante à deux points de vue: pour restaurer les caractéristiques mécaniques, mais aussi pour affiner la taille de grain. Il est acquis aujourd'hui que la déformation, la vitesse de déformation, la température de déformation, la température de maintien, et la taille de grain initiale sont des facteurs premiers qui influent sur la cinétique de Recristallisation (RX): La taille de grain joue un rôle important en tant que mesure de la surface de joint de grain disponible, sachant qu'elle agit comme site privilégié au moment de la germination. Il faut noter que peu d'études se sont intéressées au cas des très grandes tailles de grains $\left(D_{0} \approx 1 \mathrm{~mm}\right)$.

Nous présentons dans cette publication une étude des cinétiques de RXS et de Croissance de Grains (CG) d'un acier austénitique inoxydable. L'usage ultérieur de ces cinétiques sera de fournir un lot 
d'équations microstructurales susceptibles d'être utilisées lors de modélisations numériques du forgeage.

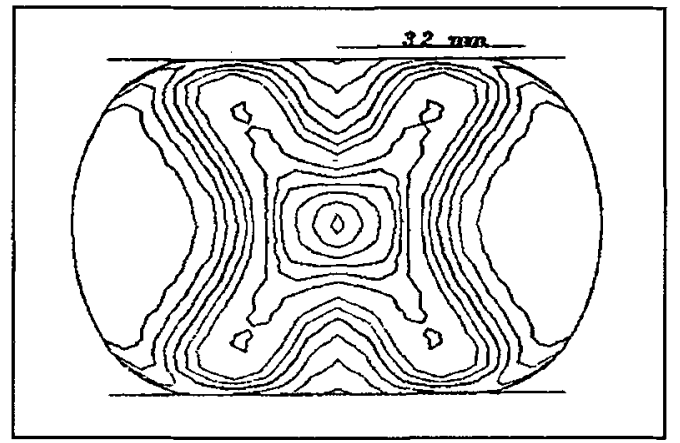

Figure 1: carte type d'isodéformations provenant du logiciel de simulation de forgeage Vulcain. Chacune des lignes représente une valeur de $\varepsilon$. L'axe orthogonal au plan de figure est parallèle aux génératrices du lingot.

\section{PROCÉDURE EXPERIMENTALE}

\subsection{Le matériau}

L'alliage expérimental est une nuance à base de fer $(\mathrm{Fe})$ comportant essentiellement: $18 \%$ en poids de chrome $(\mathrm{Cr}) ; 18 \%$ en poids de manganèse $(\mathrm{Mn}) ; 0.55 \%$ en poids d'azote $(\mathrm{N}) ;<0.1 \%$ en poids de carbone (C), plus d'autre éléments d'alliages tels que le molybdène (Mo), le vanadium (V), le nickel (Ni), etc....

Cet acier est élaboré au four électrique avec un affinage en poche sous vide (VSD), puis refondu sous laitier électroconducteur (ESR). Il en résulte une composition très fiable et très propre. La nuance est ensuite destinée a être forgée à chaud $\left(\approx 900^{\circ} \mathrm{C}\right.$ à $\left.1150^{\circ} \mathrm{C}\right)$ sous une presse de 20000 tonnes.

La structure est entièrement austénitique (Fer $\gamma$ ). C'est l'azote qui, entre autre, permet à cette nuance d'être constamment en phase $\gamma$.

Les lopins utilisés au cours de cette étude ont été prélevés au coeur de lingots commerciaux. Deux tailles de grains ont été mises à notre disposition, $1.4 \mathrm{~mm}$ et $0.7 \mathrm{~mm}$ de diamètre $\left(D_{0}\right)$. Ces microstructures dites de "départ" sont le fruit d'un très fort corroyage, destiné à briser la structure dendritique, suivi d'un recuit de 96 heures à $1200^{\circ} \mathrm{C}$, assurant une parfaite recristallisation ainsi qu'une microstructure homogène. De très grande taille moyenne de grains, ces structures sont équiaxes et ne présentent pas a priori de texture cristallographique.

\subsection{Techniques expérimentales}

\subsubsection{Recristallisation}

Les essais que nous avons effectués sont dits de "compression à chaud". Ils ont été pratiqués sous une presse expérimentale de 300 tonnes. Les lingots, de taille de grains $D_{0}=1.4 \mathrm{~mm}$ ou $D_{0}=0.7 \mathrm{~mm}$, sont des cylindres à base circulaire dont la hauteur $h$ et le diamètre $d$ sont de $8 \mathrm{~cm}$. L'écrasement des lopins, préalablement soumis à une mise à température de 1 heure $\left(T_{d e f}\right)$, s'effectue sous tas plats chauffés à $940^{\circ} \mathrm{C}$. La vitesse de déformation $(\dot{\varepsilon})$ a été estimée à $3 s^{-1}$. Des cartes d'isodéformations vraies $(\varepsilon)$, nécessaires à l'analyse des essais ont été obtenues par un code éléments finis de simulation du forgeage (Vulcain $\left({ }^{1}\right)$ ). La figure 1 représente la carte type d'une coupe transversale d'un lopin une fois déformé. Pour isoler une déformation, il suffit alors de se

\footnotetext{
1 Vulcain: code éléments finis développé à l'INSA de Lyon.
} 
référer à ces cartes, de découper un échantillon autour de la déformation recherchée et de procéder aux recuits aux températures dites températures de recuit ou températures de recristallisation $\left(T_{r e x}\right)$. La figure 2 résume le chemin suivi par chaque lopin avant d'arriver à la phase métallographique.

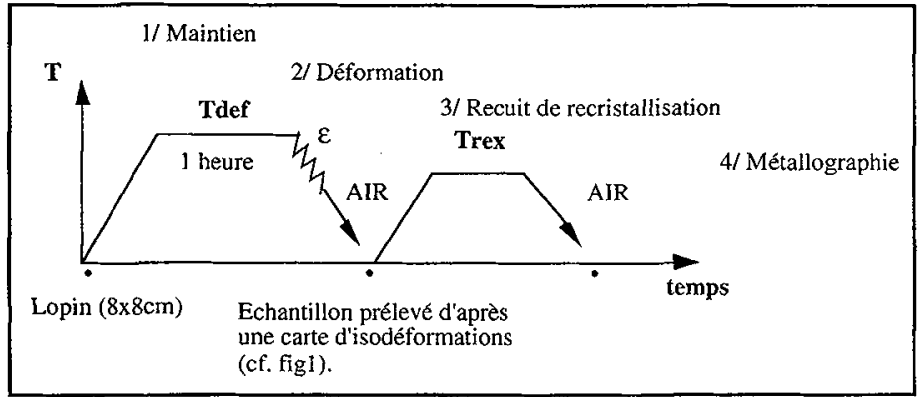

Figure 2: diagramme schématique du chemin thermomécanique $(T, t)$ type. On notera tes variables: $T_{\text {def, }} T_{\text {rex }}, \varepsilon$. La vitesse de déformation $\dot{\varepsilon}$, étant de l'ordre de $3 s^{-1}$.

Une fois le traitement thermomécanique terminé, les échantillons sont préparés en vue d'une étude des fractions recristallisées. Le polissage s'effectue de façon classique jusqu'au papier 1200, suivi d'un polissage électrolytique dans une solution d'acide perchlorique, de méthanol et de butyl-glycol $\left(2.5 \mathrm{~A} / \mathrm{cm}^{2}, 40 \mathrm{~V}\right)$. L'attaque chimique révélant joints de grains et macles de recuit utilise une solution contenant les acides nitrique, chlorhydrique et fluorhydrique, ainsi que du butyl-glycol et de l'eau. La figure 3 représente une structure en fin de cycle, typique d'une recristallisation en cours: $T_{d e f}=1100^{\circ} \mathrm{C} ; \varepsilon=0.7 ; T_{p r x}=880^{\circ} \mathrm{C}$.

La mesure des fractions recristallisées se fait de façon semi-automatique: d'après une photographie, couvrant une aire de 3 à $10 \mathrm{~mm}^{2}$, de chaque échantillon prise sur un microscope optique, on réalise un transparent soulignant les régions recristallisées. Une fois scannées, les aires recristallisées sont mesurées à l'aide d'un logiciel de traitement d'image: NIH Image $1.55\left(^{1}\right)$.

\section{2,2.1. Croissance de Grains}

A l'issue du maintien de 96 heures à $1200^{\circ} \mathrm{C}$ (cf. §2.1.), les lingots d'étude subissent un corroyage d'environ 4.0 ce qui donne un grain très fin (fig. 4). Le lopin $(8 \times 8 \mathrm{~cm})$ est ensuite découpé en cubes d'environ $1.5 \times 1.5 \times 1.5 \mathrm{~cm}$, chacun d'eux ayant été recuit à diverses températures $\left(980^{\circ} \mathrm{C}\right.$ à $\left.1200^{\circ} \mathrm{C}\right)$ et pendant des durées variables (de $15 \mathrm{~min}$. à $24 \mathrm{hrs}$.).

Afin de déterminer la taille de grains, chaque structure a été photographiée (microscopie optique), les joints de grains ont été retracés à la main sur transparents et analysés par le logiciel d'analyse d'image NIH Image 1.55 afin d'obtenir un histogramme de taille de grains à partir duquel, par interpolation avec une loi log-normale[17,18], on détermine la taille ASTM de grains.

\footnotetext{
1 NIH Imagel.55: freeware MacIntosh disponible par "anonymous ftp" sur zippy.nimh.nih.gov, développé par Wayne Rasband.
} 


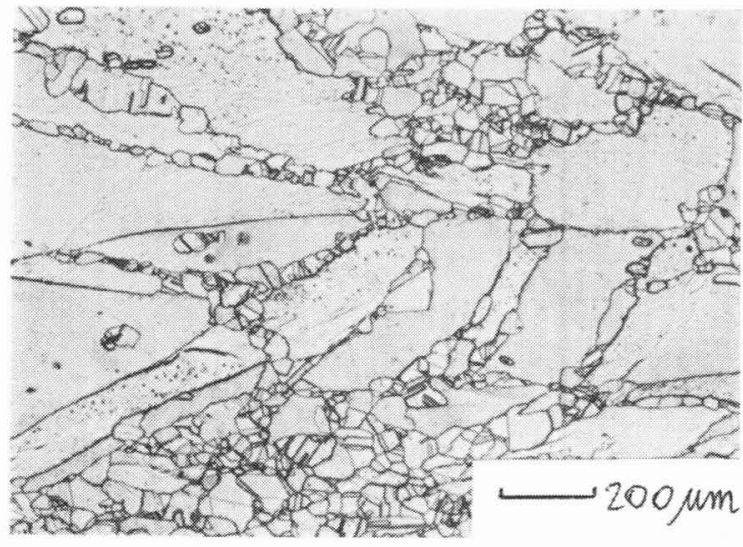

Figure 3: recristallisation statique à $880^{\circ} \mathrm{C}\left(T_{\text {rex }}\right)$ après une déformation de $70 \%(\varepsilon)$ à $1100^{\circ} \mathrm{C}\left(T_{d e f}\right)$.

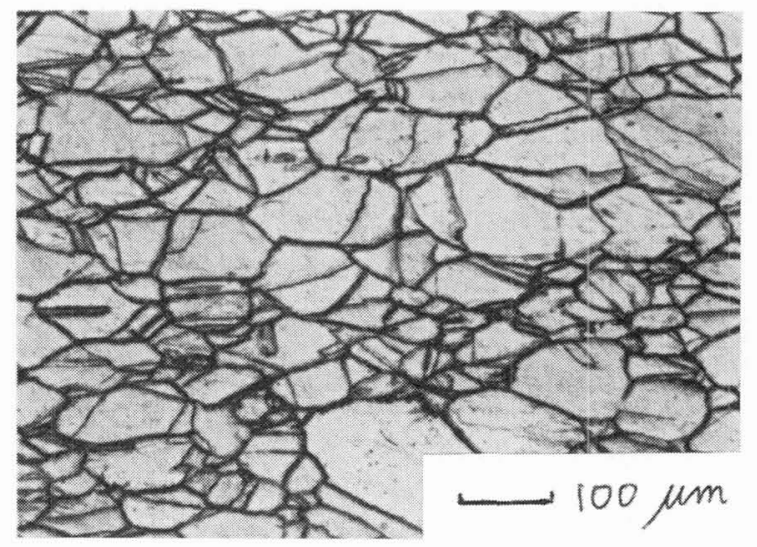

Figure 4: état initial utilisé lors des essais de CG.

\section{RÉSULTATS ET DISCUSSION}

\subsection{Recristallisation statique}

La RXS se fait essentiellement à partir des joints de grains avec quelques exceptions le long des macles de recuit [2] et dans une moindre mesure autour d'imperfections telles que les inclusions (cf. fig. 3). La séquence figure 6 et figure 7 illustre l'effet du temps de maintien sur l'évolution de la microstructure à une température constante. Le tableau 1 récapitule les différentes valeurs prises par les paramètres de l'étude. Le temps de maintien peut être contrôlé de façon assez précise, pour des temps assez courts étant donné la petite taille des échantillons recuits: cylinđres de $1 \mathrm{~cm}$ de diamètre sur $1 \mathrm{~cm}$ de hauteur environ.

Tableau 1: valeurs des différents paramètres. Se référer à la figure (2) définissant les variables $T_{\text {def }}, \mathcal{E}, T_{\text {rex }}$ et Temps. Les températures sont exprimées en "C.

\begin{tabular}{cccc}
\hline Tdef & $\mathcal{E}$ & Trex & Temps \\
\hline 940 & 0.02 & 880 & $1 \mathrm{~min}$. \\
1020 & 0.04 & 980 &. \\
1100 & 0.2 & 1060 & à \\
1180 & 0.4 & 1180 &. \\
& 0.7 & & 32 heures \\
\hline
\end{tabular}

Comme le montre le cliché de la figure 3, nous sommes en présence d'un phénomène de germination-croissance. À ce titre nous avons appliqué l'analyse faite par Melvin Avrami [3][4][5], qui d'une certaine façon, se résume à l'équation suivante:

$$
X=1-\exp \left(\beta \cdot t^{k}\right)
$$

où $X$ est la fraction volumique recristallisée, supposée égale à la fraction surfacique mesurée $\left({ }^{1}\right), \beta$ une constante et $k$, l'exposant d'Avrami.

1 Vrai dans le cas d'une structure totalement équiaxe. 
En théorie, $k$ prend des valeurs discrètes entières comprises entre 1 et 4 . 1 correspondant à une croissance linéaire alors que 4 serait attribuée à une croissance tridimensionnelle [4]. Ici, nous avons utilisé cette équation de façon classique pour interpoler nos mesures de $X$. L'interpolation linéaire de pente $k$ se fait immédiatement pour peu que l'on se place dans un système de "coordonnées d'Avrami", à savoir: $X=\log (t)$ et $Y=\log [\ln (1 /(1-X)]$. Nous avons de cette façon mesuré 21 valeurs de $\mathrm{k}$ comprises entre 0.3 et 1.0. Les valeurs de l'ordre de 0.4 se rencontrent aux températures élevées de déformation, par exemple $k=0.3$ pour $\varepsilon=0.04, T_{\text {def }}=1180^{\circ} \mathrm{C}$ et $T_{\text {rex }}=1180^{\circ} \mathrm{C}$. Malgré cet écart apparent nous avons choisi de fixer $\mathrm{k}$ à 1 qui est une valeur assez répandue dans les études de ce type. De tels écarts par rapport à l'étude d'Avrami s'expliquent à la fois de façon expérimentale et de manière numérique. Rollet et al.[19] ont montré par simulation Monte-Carlo qu'une hétérogénéité de déformation à l'intérieur des grains pouvait être à l'origine de coefficients nettement en deçà de 1 . Ils ont aussi montré la possibilité d'obtenir une variation de $k$ au cours de la transformation. Barraclough et Sellars[7] nous donnent l'explication suivante: les dislocations ayant tendance à s'empiler sur les joints de grains, il se crée naturellement un gradient de densité de dislocations décroissant du bords vers le centre des grains; la vitesse de migration $G$, du front recristallisant étant directement liée à cette densité, au fur et à mesure que le front s'éloigne du joint de grain originel, $G$ diminue entrainant un ralentissement de la cinétique.

D'un point de vue purement mathématique remarquons qu'il n'existe de point d'inflexion (éq. 1) que pour les valeurs de $k$ supérieures strictement à 1 . En deçà de cette valeur la courbe $(X, t)$ de l'équation 1 n'est plus de forme "sigmoïdale" ce qui est plus conforme au cas des courbes expérimentales (cf. fig. 5).

Vient ensuite une analyse de la constante $\beta$. Avrami [3] a montré dans son article qu'il suffit de résoudre le problème de la recristallisation pour un temps caractéristique $\tau$ à partir duquel toute transformation en temps réel $t$ se déduit d'un simple changement d'échelle. Entre d'autres termes, par un changement d'échelle de temps, tous les points des différentes transformations isothermes doivent s'aligner sur une même courbe. En prenant $t_{0.5}$, on obtient pour $\beta$ :

$$
\beta=\ln (0.5) / t^{k}{ }_{0.5}
$$

$t_{0.5}$ est ensuite interpolé par l'équation suivante (C.M. Sellars [7]):

$$
t_{0.5}=\alpha \cdot \varepsilon^{a} \cdot D_{0}^{b} \cdot Z^{c} \cdot \exp \left(Q_{r e x} / R T\right)
$$

où $\alpha$ est une constante, $D_{0}$ la taille de grains de départ, $\varepsilon$ la déformation vraie, $Z$ le coefficient de Zener Hollomon faisant intervenir une loi d'Arrhenius et le taux de déformation $\dot{\varepsilon}$ selon une loi puissance, $Q_{\text {rex }}$, l'énergie d'activation et T la température du recuit isotherme pratiquée. $\alpha, a, b$, $Q_{t l f}$ et $Q_{r x x}$ ont été déterminés par une interpolation linéaire à 4 inconnues sur les paramètres: $T_{d t f}$, $T_{r e x}$, $\varepsilon$ et $D_{0}$.

La figure 5 illustre une confrontation entre deux courbes expérimentales correspondant à deux températures de recuit différentes (les autres paramètres étant égaux par ailleurs) et deux courbes théoriques provenant des équations 1,2 et 3 avec les valeurs présentées dans le tableau 2. L'exposant d'Avrami est égal à 1 et on remarquera que ces courbes ne sont pas de forme sigmoïdale. Bien que l'interpolation soit assez bonne, les hypothèses ayant permis à Avrami d'obtenir un exposant de 1 ne sont pas vérifiées. Il faut donc considérer cette méthode d'analyse plus empirique qu'analytique. 


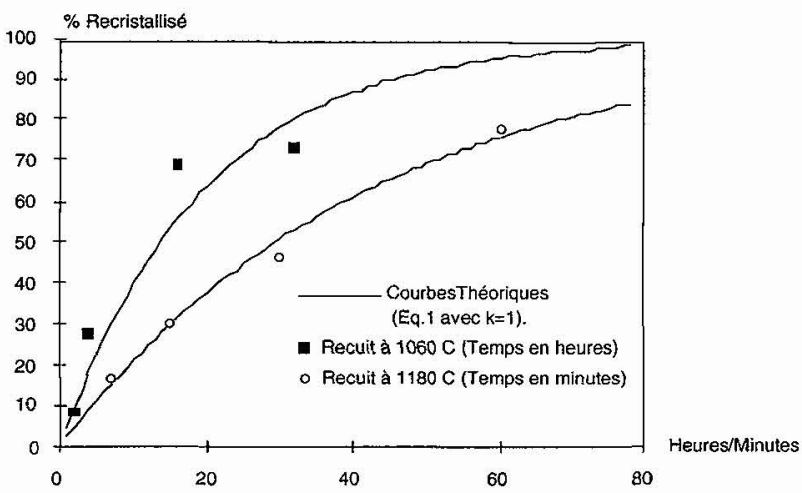

Figure 5: comparaison des courbes théoriques et expérimentales, fraction recristallisée (\%) versus temps, pour deux températures de recuit $\left(T_{\text {rex }}\right) . T_{\text {def }}=1020^{\circ} \mathrm{C}, \varepsilon=0.04, D_{0}=1.4 \mathrm{~mm}$ et $T_{\text {rex }}$ égale à $1060^{\circ} \mathrm{C}$ et $1180^{\circ} \mathrm{C}$. Les coefficients de l'équation 1 sont ceux du tableau 2 .

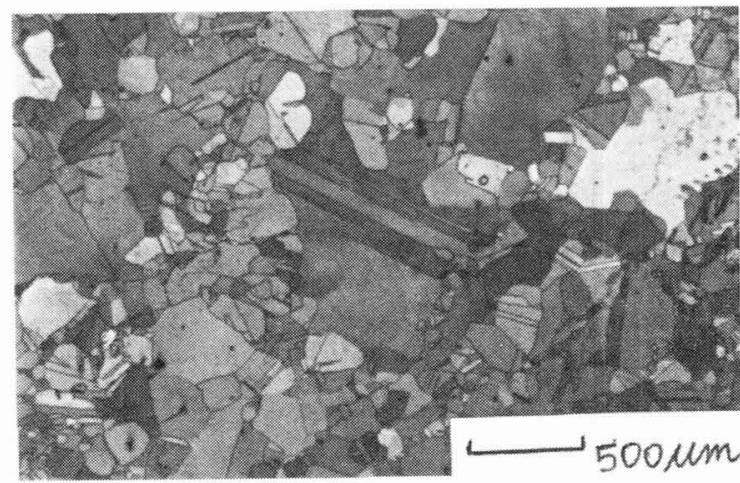

Figure 6: aspect de la microstructure après un cycle: $T_{\text {def }}=1020^{\circ} \mathrm{C} ; \varepsilon=0.2 ; T_{r e x}=980^{\circ} \mathrm{C}$, pour un temps de maintien de 1 heure. La fraction recristallisée de cet état est de $73 \%$.

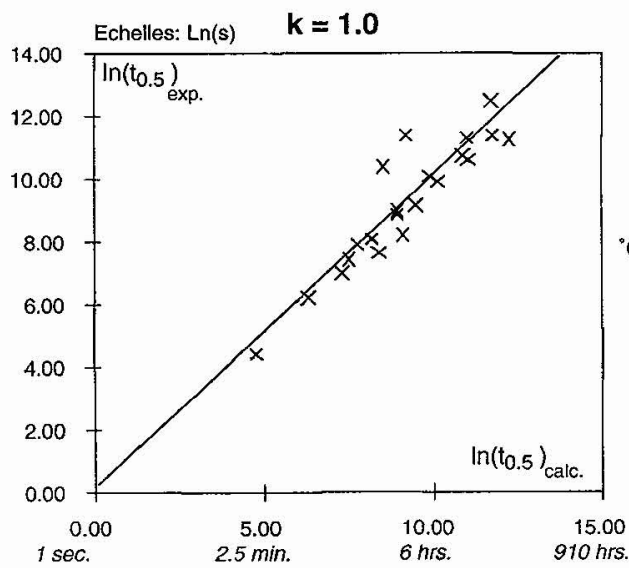

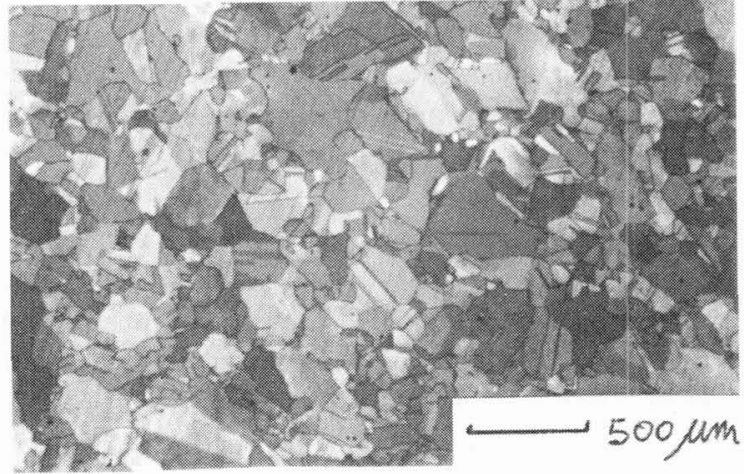

Figure 7: aspect de la microstructure après un cycle: $T_{\text {def }}=1020^{\circ} \mathrm{C} ; \varepsilon=0.2 ; T_{r e x}=980^{\circ} \mathrm{C}$, pour un temps de maintien de 2 heures. La fraction recristallisée de cet état est de $82 \%$.

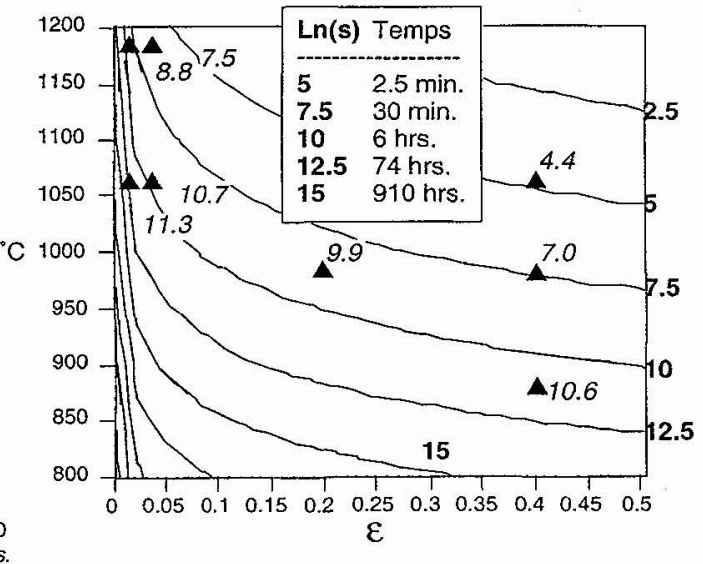

Figure 9: tracé des iso- $\ln \left(t_{0.5}\right)$ en fonction de

Figure 8: confrontation expérience-calcul. Axe des $X$ : résultats du calcul de $t_{0.5}$ (s) (3); axe des $Y$ : résultats expérimentaux.
$T_{\text {rux }}$ et de $\varepsilon$, pour $D_{0}=0.7 \mathrm{~mm}$ et

$T_{d e f}^{\text {rex }}=1020^{\circ} \mathrm{C}$. D'après l'équation (3). Les triangles représentent quelques points expérimentaux. 
Les valeurs des paramètres de l'équation (3) sont données dans le tableau 2, ainsi qu'une analyse statistique. Cette analyse porte sur les 21 mesures de $\beta\left(t_{0.5}\right)$. On notera que le coefficient de corrélation $\left(r^{2}\right)$, est ici d'une valeur de 0.85 , signe d'une bonne corrélation. Deux tests ont été employés pour verifier, analyser et justifier l'emploi des quatre paramètres: $T_{\text {def }}, T_{r e x}, \varepsilon$ et $D_{0}$.

- Le test de Student est employé dans le cas d'une analyse linéaire lorsque l'on pose comme hypothèse que la distribution de chacun des estimateurs est normale. Dans ce cas, intervalle de confiance et niveau de confiance proviennent des lois de Student et de Fischer. "t-obs" est le rapport de la mesure de la valeur moyenne à la mesure de la variance. "t-test" est la valeur théorique de ce ratio pour un niveau de confiance donné et un nombre de degrés de liberté donné. "t-test" se lit dans des tables numériques donnant les lois de Student.

Ce test s'analyse de la façon suivante: on compare les valeurs mesurées, t-obs, à celle de Student, ttest. On en deduit alors si la variable $\mathrm{x}_{\mathrm{i}}$ est influante ( $\mathrm{t}$-obs $>\mathrm{t}$-test) et son degré d'influence par rapport aux autres variables.

- Le test-F (de Fischer) est employé dans les mêmes hypothèses que le test de Student pour determiner si le modèle linéaire est bien judicieux. Dans ce cas F-obs $>$ F-test. Ce test est basé sur une loi de Fischer, d'où son nom.

On se réfèrera à tout ouvrage sur les méthodes usuelles de statistique pour une démonstration rigoureuse.

L'ordre d'influence est le suivant, en partant du plus influent: $T_{\text {rex }}, \varepsilon, T_{\text {thf }}, D_{0}$ (comparer la valeur de t-obs à celle de t-test). La température de déformation est quasi-négligeable et la taille moyenne de grains (dans notre plage d'essai) ne présente pas d'influence significative.

Tableau 2: valeurs calculées à partir des données expérimentales des coefficients de l'équation (3). L'influence de chacun des paramètres se fait en comparant les valeurs de t-test et t-obs. $Q_{\text {rex }}$ et $Q_{\text {def }}$ sont exprimées en $\mathrm{kJ} / \mathrm{mol}$. a et b sont sans dimension. $\alpha$ est de dimension: $s^{1} \mathrm{~mm}^{-b}$.

\begin{tabular}{ccccccc}
\hline & Qdef & Qrex & $b$ & $a$ & $\alpha$ \\
\hline Valeurs & -159017 & 446249 & 1.88 & -2.04 & 3.30 E-10 \\
t-obs & 3.03 & 8.20 & 2.17 & 7.05 & 3.63 \\
\hline t-test & 2.57 & & & & \\
$r^{\wedge} 2$ & 0.85 & & & & \\
F-obs & 22.72 & & & & \\
F-test & 2.85 & & & & & \\
\hline
\end{tabular}

La figure 9 est une illustration d'iso- $\operatorname{In}\left(t_{0.5}\right)$ obtenues par l'équation (3) en fonction de la température de maintien et de la déformation introduite. Noter l'accord satisfaisant entre points expérimentaux et iso-valeurs calculées. On remarque aussi un tassement des lignes aux faibles valeurs de la déformation. Il existe à l'intérieur de ce graphe une fenêtre où la recristallisation n'est pas possible en temps raisonnable (i.e. $<60 \mathrm{hrs}$ ). Schématiquement on ne peut pas espérer faire recristalliser le matériau pour des valeurs de $T_{r e x}$ inférieures à $880^{\circ} \mathrm{C}$ ou des déformations inférieures à $2 \%$.

D'autre part, il existe, une limite supérieure au delà de laquelle augmenter la déformation ne diminue plus le temps de recristallisation [6]. Il a été trouvé pour les acier de type 316L une limite aux environs de $60 \%$. Notre étude s'intéressant particulièrement aux faibles déformations, nous n'avons pas mis en évidence une telle limite.

Les énergies d'activations de $446 \mathrm{~kJ} / \mathrm{mol}$ pour $Q_{\text {rex }}$ sont supérieures à celles qui sont citées dans la littérature où généralement on fait état de valeurs aux alentours de $300 \mathrm{~kJ} / \mathrm{mol}$ pour les aciers de type $316,316 \mathrm{~L}$ ou 304 . On notera toutefois une valeur de $425 \mathrm{~kJ} / \mathrm{mol}$ donnée par Barraclough et Sellars 
[7]. L'étude de Hinojosa et al. [20] sur les énergies d'activation employées dans les équations d'Avrami modifiées par Sellars fait apparaître un rapport de l'ordre de 0.75 à 0.95 entre Qdef et Qrex. Ce que nous retrouvons dans notre étude si on prend c (éq.3) de l'ordre de 0.5 qui est une valeur couramment employée. D'autre part ces mêmes auteurs font apparaître une énergie d'autodiffusion de l'austénite de $283 \mathrm{~kJ} / \mathrm{mol}$ qui est fonction de la composition chimique. Cette valeur a été déterminée sur des aciers bas carbone. Il semblerait d'après ces auteurs que cette valeur augmente avec les teneurs en manganèse et carbone. Nous voyons donc que nos mesures, bien qu'élevées, sont d'un ordre de grandeur raisonnable.

\subsection{Croissance de Grains}

44 essais de recuits thermiques isothermes ont été réalisés et analysés selon une loi du type:

$$
A=A_{0}+\alpha \cdot \exp (Q / R T) \cdot t^{n}
$$

où $A$ est l'aire moyenne des grains, $A_{0}$ l'aire moyenne initiale (juste en fin de recristallisation), $\alpha$ une constante, $Q$ une énergie d'activation, $t$ le temps et $n$ une constante.

Nous avons choisi de représenter les aires moyennes plutôt que les diamètres moyens pour s'affranchir d'une approximation supplémentaire qui serait de considérer les grains comme sphériques. En effet les tailles moyennes ont été déterminées par une mesure directe de la surface de chacun des grains et non pas par une mesure du type "intersection de lignes" qui elle aurait donné une longueur moyenne proportionnelle au diamètre équivalent du grain. D'ordinaire il est souvent utilisé une loi faisant intervenir les diamètres moyens plutôt que les aires car la plupart des théories de croissance normale de grain s'appuient sur les diamètres équivalents, mais il ne semble pas y avoir de justification théorique à utiliser l'un plutôt que l'autre. D'autre part la taille initiale étant souvent faible on peut alors la négliger et les deux formulations (aires ou diamètres) deviennent équivalentes.

La taille de grains initiale est souvent considérée comme un paramètre d'ajustement.

Nous avons choisi une valeur de $100 \mathrm{\mu m}^{2}$. L'exposant $n$ prend de très nombreuses valeurs selon les études, qu'elles soient numériques $[9,10,11]$, analytiques [12,13] ou purement expérimentales[14]. Nous avons donc, contrairement au cas de la recristallisation où l'exposant a été fixé par principe, décidé de considérer $n$ comme un paramètre d'ajustement. La fenêtre temps-température balayée va de 15 minutes à 24 heures et de $980^{\circ} \mathrm{C}$ à $1200^{\circ} \mathrm{C}$. Les résultats de l'interpolation linéaire à 2 variables (temps, température) sont présentés dans le tableau 3 et sur la figure 10 où se confrontent les tailles de grains ASTM $[1,16]$ mesurées et calculées. On remarque une très bonne corrélation des variances (F-test) et l'importance première de la température.

La valeur de $\mathrm{n}$ est de 0.6 ou de 0.3 si on passe à une description en terme de diamètres moyens. D'après la revue bibliographique d'Atkinson[15] sur le sujet, la valeur la plus communément mesurée expérimentalement est $1 / 3$ soit une valeur très proche de celle de notre étude. Les études théoriques mettent en général en avant une valeur de 0.5 pour le cas de la croissance normale de grain pure, c'est à dire ne faisant pas intervenir par exemple de retardement dû à la précipitation ou aux inclusions. Rien ne permet aujourd'hui d'affirmer que 0.5 est bien la valeur que l'on obtiendrait avec un matériau tout à fait pur ni pourquoi les études expérimentales s'éloignent de ce cas idéal. Il faut se rendre compte qu'il existe un grand nombre de théories et que certaines, comme celle de Louat [21], (1974) qui reproduisent assez correctement la réalité n'ont toujours pas reçu de justification physique acceptable (cf. Atkinson [15]). Il faut donc surtout considérer ces équations comme une approche semi-empirique du problème et recalculer les paramètres $(Q, n$ et $\alpha)$ pour chaque matériau étudié. 
Comme nous le montre l'illustration de la figure 11, il est possible de définir une fenêtre de taille de grains ASTM par un contrôle de la température et du temps de maintien.

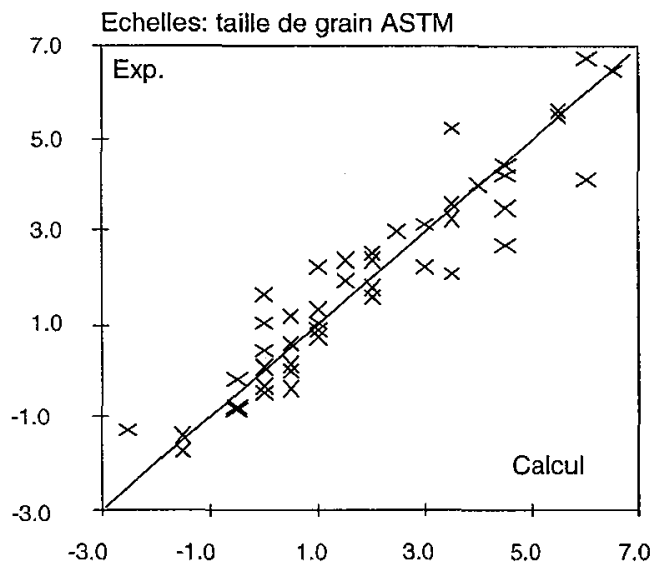

Figure 10: confrontation expérience-calcul des tailles de grains ASTM mesurées et calculées par l'équation (4).

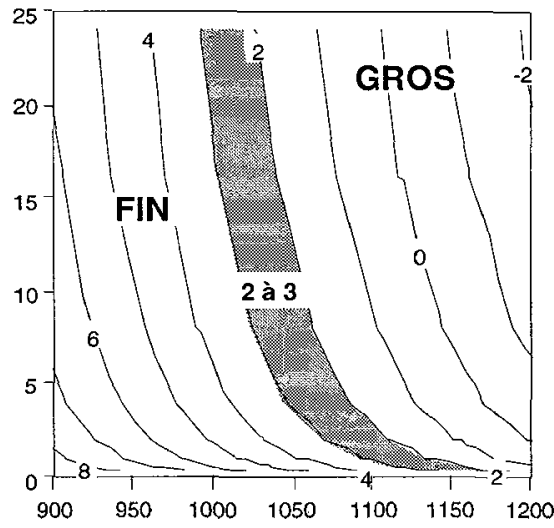

Figure 11: carte des iso-tailles de grains ASTM en fonction de la température $\left({ }^{\circ} \mathrm{C}\right)$ et du temps (hrs.) de maintien. D'après l'équation (4).

Tableau 3: valeurs des paramètres de l'équation (4) et analyse statistique de 44 essais de croissance de grains. $Q$ est exprimée en $\mathrm{kJ} / \mathrm{mol}$. $\alpha$ en $s^{-n} \mu \mathrm{m}^{2}$. Pour l'interprétation des tests statistiques voir la discussion au chapitre précédant.

\begin{tabular}{cccc}
\hline & $Q$ & $n$ & $\alpha$ \\
\hline Valeurs & -268091 & 0.60 & $1.4326 \mathrm{E}+12$ \\
t-obs & 14.12 & 11.57 & 16.53 \\
\hline t-test & 2.57 & & \\
$r^{\wedge} 2$ & 0.88 & & \\
F-obs & 160.52 & & \\
F-test & 2.44 & & \\
\hline
\end{tabular}

\section{CONCLUSION}

1. Les courbes de RXS de l'acier austénitique inoxydable étudié à l'aide d'essais de compression à chaud nous ont permis de mettre en évidence une cinétique conforme à celle d'Avrami. Néanmoins, nous pensons qu'il n'y a pas de lien entre les hypothèses théoriques d'Avrami et les phénomènes réels mis en jeu lors de la RXS. Plus qu'une croissance linéaire, l'hétérogénéité de la répartition de la déformation au sein de chaque grain (surtout lorsque la structure a une taille de grains très élevée) serait la cause de coefficients $k$ proches et inférieurs à 1 .

2. Le temps de mi-recristallisation a été mis en équation sous forme de loi puissance. Un accord satisfaisant a été réalisé.

3. La CG se fait selon une loi du type $A=A_{0}+\alpha \cdot \exp (Q / R T) t^{n}$ pour laquelle nous avons trouvé un exposant de 0.6. Notons que cette valeur est très proche de celles de nombreuses autres études sur ce même sujet. 
4. La prochaine étape sera d'utiliser ces équations dans un code de simulation du forgeage pour pouvoir prédire la taille de grains finale.

\section{BIBLIOGRAPHIE}

[1] ASTM Standards, vol. 03.01, pp. 297-322, 1993.

[2] Wadsworth J. E. J., Sellars C. M., Recrystallization '92, Materials Science Forum, vol. 113-115, pp. 417423, 1990.

[3] Avrami Melvin, Journal of Chemical Physics, vol. 7, pp. 1103-1112, 1939.

[4] Avrami Melvin, Journal of Chemical Physics, vol. 8, pp. 212-224, 1940.

[5] Avrami Melvin, Journal of Chemical Physics, vol. 9, pp. 177-184, 1941.

[6] Barbosa R. A. N. M. and Sellars C. M., Recrystallization '92, Materials Science Forum, vol. 113-115, pp. 461-466, 1993.

[7] Barraclough D. R. and Sellars C. M., Metal Science, pp. 257-267, 1979.

[8] Hillert M., Acta Metallurgica, vol. 13, pp. 227-238, 1965.

[9] Novikov V. Y., Acta Metallurgica, vol. 26, pp. 1739-1744, 1978.

[10] Novikov V.Y., Acta Metallurgica, vol. 42, pp. 1639-1642, 1994.

[11] Matsuura K., Itho Y., ISIJ International, vol. 31, pp. 366-371, 1991.

[12] Rhines F. N., Craig K. R., DeHoff R., Metallurgical Transactions, A, vol. 5, pp. 413-425, 1974.

[13] Fortes M.A., Acta Metallurgica, vol. 42, pp. 1643-1651, 1994.

[14] Kurtz S.K., Carpay F.M.A, Journal of Applied Physics, vol. 51, pp. 5745-5754, 1981.

[15] Atkinson H. V., Acta Metallurgica, vol. 36, pp. 469-491, 1988.

[16] Vander Voort G. F. and Friel J. J., Materials Characterization, vol. 29, pp. 293-312, 1992.

[17] Nunez C. and Domingo S., Metallurgical Transactions A, vol. 19A, pp. 2937-2944, 1988.

[18] Okazaki K., Conrad H., Transactions JIM, vol. 13, pp. 198-204, 1972.

[19] Rollet A. D., Srolovitz D. J., Doherty R. D. and Anderson M. P., Acta Metallurgica, vol. 37, pp. 627639, 1989.

[20] Hinojosa M., Ortiz U., and Colas R., Recrystallization '92, vol. 113-115, pp. 467-472, 1993.

[21] Louat N. P., Acta Metallurgica, vol. 22, pp. 721-724, 1974. 